\title{
PTEN modulates neurites outgrowth and neuron apoptosis involving the PI3K/Akt/mTOR signaling pathway
}

\author{
SHEN LIU ${ }^{1 *}$, JUN JIA $^{2^{*}}$, HENGXING ZHOU $^{1 *}$, CHI ZHANG $^{1 *}$, LU LIU $^{1}$, JUN LIU $^{1,3}$, LU LU $^{1}$, XUEYING LI ${ }^{4}$, \\ YI KANG $^{1}$, YONGFU LOU ${ }^{1}$, ZHIWEI CAI ${ }^{1}$, YIMING REN ${ }^{1}$, XIAOHONG KONG ${ }^{5}$ and SHIQING FENG ${ }^{1}$ \\ ${ }^{1}$ Department of Orthopedics, Tianjin Medical University General Hospital, Tianjin 300052; \\ ${ }^{2}$ Department of Trauma Orthopedics, Tianjin Hospital, Tianjin 300211; ${ }^{3}$ Department of Orthopedics, The First Affiliated \\ Hospital of Gannan Medical University Hospital, Ganzhou, Jiangxi 341000; ${ }^{4}$ Key Laboratory of Immuno Microenvironment \\ and Disease of the Educational Ministry of China, Department of Immunology, Tianjin Medical University, Tianjin 300070; \\ ${ }^{5}$ Laboratory of Medical Molecular Virology, School of Medicine, Nankai University, Tianjin 300071, P.R. China
}

Received December 16, 2018; Accepted July 18, 2019

DOI: $10.3892 / \mathrm{mmr} .2019 .10670$

\begin{abstract}
The present study aimed to explore the role of the PTEN/Akt/mTOR signaling pathway in the neurite outgrowth and apoptosis of cortical neurons. Cortical neurons were seeded on or adjacent to chondroitin sulfate proteoglycans. The length, number and crossing behavior of the neurites were calculated. Immunohistochemical staining and TUNEL data were analyzed. Neurites treated with PTEN inhibitor exhibited significant enhancements in elongation, initiation and crossing abilities when they encountered chondroitin sulfate proteoglycans in vitro. These effects disappeared when the PTEN/Akt/mTOR signaling pathway was blocked. Neurons exhibited significant enhancements in survival ability following PTEN inhibition. The present study demonstrated that PTEN inhibition can promote axonal elongation and initiation in cerebral cortical neurons, as well as the ability to cross the chondroitin sulfate proteoglycan border. In addition, PTEN inhibition is useful for protecting the neuron from apoptosis. The PTEN/Akt/mTOR signaling pathway is an important signaling pathway.
\end{abstract}

Correspondence to: Professor Shiqing Feng, Department of Orthopedics, Tianjin Medical University General Hospital, 154 Anshan Road, Heping, Tianjin 300052, P.R. China

E-mail: sqfeng@tmu.edu.cn

Professor Xiaohong Kong, Laboratory of Medical Molecular Virology, School of Medicine, Nankai University, 94 Weijin Road, Nankai, Tianjin 300071, P.R. China

E-mail:kongxh@nankai.edu.cn

*Contributed equally

Key words: axon growth/regeneration, apoptosis, PTEN/Akt/mTOR signaling pathway, spinal cord injury

\section{Introduction}

Spinal cord injury (SCI) is a severe and traumatic disease; due to of the high rates of disability and fatality, it results in a series of personal and social problems. The data of the National SCI database show that the current prevalence of SCI in the US has already reached 63,109 patients, with an estimated annual incidence of $\sim 54$ cases per million in 2012 (1). In addition, functional deficits always accompany a SCI, and only $0.4 \%$ of patients experience complete neurological recovery. This is due to the fact that axons fail to regenerate after SCI (2-4). This failure to regenerate can be attributed to two causes: Mature neurons exhibiting a low intrinsic ability to regrow axons and the extrinsic environment determines the projection of the axon regeneration after SCI (5-11). In addition, mature neurons have a low intrinsic ability to regrow axons $(12,13)$. In past decades, a number of studies $(2,14,15)$ have focused on characterizing environmental inhibitory molecules in the adult central nervous system. However, blocking the inhibitory activities of chondroitin sulfate proteoglycans (CSPGs) and myelin-associated molecules in the glial scar via either genetic or pharmacological means allows for the regrowth of some injured axons, even though their regeneration is limited $(14,15)$. This evidence suggests that removing inhibitory activities alone is not sufficient to allow the majority of injured axons to regenerate and gain adequate functional recovery. It is important to understand the underlying mechanisms of both the diminished intrinsic growth and axon regenerative abilities of neurons.

Numerous studies $(13,16-18)$ have indicated that manipulating certain signaling pathways, including the PTEN/mTOR, Janus kinase/STAT, dual leucine zipper kinase/JNK and suppressor of cytokine signaling 3/STAT3 pathways, may allow certain populations of mature central nervous system (CNS) neurons to mount regenerative growth after injury. However, the primary cellular substrate of PTEN is the second messenger phosphatidylinositol $(3,4,5)$-trisphosphate (PIP3), which transmits growth and survival signals (16). PTEN removes the D3 phosphate from PIP3, inactivating the $\mathrm{PI} 3 \mathrm{~K} / \mathrm{Akt} / \mathrm{mTOR}$ pathway and generating phosphatidylinositol $(4,5)$-bisphosphate (PIP2), which does not function in the same 
manner as PIP3 (19-21). In a previous study, Park et al (22) found that the regeneration and survival abilities of a retinal ganglion cell with PTEN gene knockdown were superior to those of normal retinal ganglion cells, and were correlated with the content of mTOR. Our previous study (23) also demonstrated that PTEN silencing using short hairpin RNA (shRNA) promoted neurite elongation and motor function improvement in a rat model of SCI.

In the present study, an inhibitory microenvironment of SCI was constructed in vitro. An inhibitor with a high inhibition efficiency targeted against the PTEN/mTOR signaling pathway was used to explore the mechanism of axon growth/regeneration promotion. As PTEN also affects apoptosis in a number of cell types, the effects of PTEN on neuronal apoptosis were also explored.

\section{Materials and methods}

Animal subjects and ethics statement. A total of 24 new born Wistar rats (5-6 g) were provided by the Radiation Study Institute-Animal Center at Tianjin Medical University. All experimental procedures involving animals were approved by the Ethics Committee of Tianjin Medical University and strictly complied with the-Ethical Principles for the Maintenance and Use of Animals In Neuroscience Research (24).

Neuron isolation and culture. In brief, forebrain cortices from postnatal day 0 (P0) Wistar rats were dissected under a stereomicroscope (LEICA M501; Leica Microsystems GmbH) and dissociated into a single-cell suspension through enzymatic digestion (Papain and DNase I; Worthington Biochemical Corporation) and mechanical pipetting. After centrifugation for $5 \mathrm{~min}$ at $200 \mathrm{x} \mathrm{g}$ and $4^{\circ} \mathrm{C}$, the cells were resuspended at a density of $6 \times 10^{5}$ cells $/ \mathrm{ml}$ in fresh plating medium [DMEM-high containing 10\% FBS (both Gibco; Thermo Fisher Scientific, Inc.) and $1 \%$ (vol/vol) penicillin/streptomycin (Sigma-Aldrich; Merck KGaA)]. The cells were cultured in culture plates (BD Falcon; BD Biosciences) coated with $0.01 \%$ poly-L-lysine (PLL; Sigma-Aldrich; Merck $\mathrm{KGaA}$ ) at $37^{\circ} \mathrm{C}$ in a humidified incubator with $5 \% \mathrm{CO}_{2}$. The plating medium was replaced by serum-free medium [Neurobasal containing $10 \mathrm{ng} / \mathrm{ml}$ neuronal growth factor, $2 \%$ (vol/vol) B27 supplement, $0.5 \mathrm{mM}$ L-glutamine (all Gibco; Thermo Fisher Scientific, Inc.), $0.5 \%$ (vol/vol) D-glucose and $0.5 \%$ (vol/vol) penicillin/streptomycin (Sigma-Aldrich; Merck KGaA)] 4 h later. Half of the serum-free medium was replaced every 3 days. A primary antibody against $\beta$-tubulin III (1:500; Abcam, ab18207) was applied as a specific axonal marker to identify the neurons. In addition, Hoechst 33342 ( $1 \mu \mathrm{g} / \mathrm{ml}$; Invitrogen; Thermo Fisher Scientific, Inc.) was used to visualize the nuclei of all cells in TUNEL staining.

Preliminary specific inhibitors efficiency assay. The inhibitor of PTEN dipotassium bisperoxo (picolinato) oxovanadate [bpV(pic); Sigma-Aldrich; Merck KGaA] was reconstituted in $\mathrm{ddH}_{2} \mathrm{O}$ for a $500-\mu \mathrm{M}$ stock; different concentrations $(100,300,500$, and $700 \mathrm{nM})$ were tested (data not shown) and the final concentration used was $500 \mathrm{nM}$. Inhibitive efficiency of $\mathrm{bpV}$ (pic) was still lower than that of control group at day 14 (data not shown). The highly selective inhibitor of PI3K LY294002 (Cell Signaling Technology, Inc.) was reconstituted in DMSO for a 10-mM stock; the final concentration used was $50 \mu \mathrm{M}$. The inhibitor of mTOR ridaforolimus (Santa Cruz Biotechnology, Inc.) was reconstituted in DMSO for a $100-\mu \mathrm{M}$ stock; the final concentration used was $100 \mathrm{nM}$. To evaluate the efficiency of the inhibitors of the PTEN/Akt/mTOR signaling pathway, the neurons were separated into four treatment groups [control, LY294002 + bpV(pic), ridaforolimus + bpV(pic) and $\mathrm{bpV}(\mathrm{pic})]$. Half of the culture medium was replaced every 3 days. These samples were collected for western blot analysis at day 7 , based on a phosphorylation pattern study. In addition, primary antibodies for Akt (cat. no. 4691, 1:1,000), phosphorylated (p-)Akt (cat. no. 4060, 1:1,000), mTOR (cat. no. 2983, 1:1,000), p-mTOR (cat. no. 5536, 1:1,000), p70-S6 kinase 1 (p70S6K; cat. no. 97596, 1:1,000) and p-p70S6K (cat. no. 97596, 1:1,000; all Cell Signaling Technology, Inc.) were used in this procedure at $4^{\circ} \mathrm{C}$ overnight.

Plating preparation. To explore the effect of specific inhibitors on axonal growth, 6-well plates were coated with $0.01 \%$ PLL overnight. The next day, they were washed three times with PBS and dried at $37^{\circ} \mathrm{C}$. Then, 3- $\mu 1$ droplets of CSPGs (50 $\mu \mathrm{g} / \mathrm{ml}$; EMD Millipore) were spotted onto the 6 -well plates for $4 \mathrm{~h}$ at $37^{\circ} \mathrm{C}$. After the droplets dried, the six-well plates were covered with laminin(LN) $(10 \mu \mathrm{g} / \mathrm{ml}$; Invitrogen) for $2 \mathrm{~h}$ at $37^{\circ} \mathrm{C}$. They were then washed three times with PBS and stored at $37^{\circ} \mathrm{C}$ before neuron plating.

Immunocytochemistry. Immunocytochemistry was performed as described previously (25). Neurons were treated with the various inhibitors for 3 days, then the medium was changed. Cytochemistry was performed on day 7 , and all cytochemistry was performed on cells cultured on CSPGs-coated plates. After 10 days of culture, the four groups of neurons were fixed with 4\% PFA in PBS for 5 min and incubated in $0.3 \%$ Triton X-100 (Sigma-Aldrich; Merck KGaA) for 5 min and blocking solution containing $10 \%$ goat serum (OriGene Technologies, Inc.) in PBS for $60 \mathrm{~min}$ all these steps are at room temperature. The primary antibodies, mouse (IgM) anti-CSPG (clone CS-56; 1:500; Sigma-Aldrich; Merck KGaA, Cat no. SAB2100493) and rabbit anti- $\beta$-tubulin III (1:100; Abcam, Cat no. ab18207), were used to identify CSPGs and neurons, respectively. All cultures were stained with primary antibodies overnight at $4^{\circ} \mathrm{C}$. The cultures were then washed three times and stained with the secondary antibodies AMCA-conjugated goat anti-mouse IgM (Jackson ImmunoResearch cat. no. 115-155-020) and TRITC-conjugated goat anti-rabbit IgG (OriGene Technologies, Inc. cat. no. S0015) for $1 \mathrm{~h}$ at room temperature. Hoechst 33342 was used to visualize the nuclei for $10 \mathrm{~min}$ at room temperature.

Quantification of neurites. Neurite length and the extent of branching were calculated from images of at least 120 neurons for each culture condition that were acquired under a fluorescence microscope (magnification, x20; IX71; Olympus Corporation). Neurites extending over the CSPG substrate were traced and measured using the Image-Pro Plus 7.0 software (Media Cybernetics, Inc.) for each field. Maximum neurite length was defined as the length of the longest continuous neurite. The number of primary neurites was defined as the number of neurites that were extending from the cell body. In light of the enhanced crossing ability onto 
CSPGs, only individually identified axons were analyzed, and the percentage of neurites that crossed the CSPGs border was calculated.

Western blot analysis. After 7 days, the cells were placed for $30 \mathrm{~min}$ in culture medium containing a final concentration of $100 \mu \mathrm{M} \mathrm{H}_{2} \mathrm{O}_{2}$ at $37^{\circ} \mathrm{C}$ to induce apoptosis of neurons. Western blotting analysis was performed as described previously (26), with minor modifications. The total protein of each treatment group was extracted from neurons on the $10-\mathrm{cm}$ dishes using RIPA buffer (Santa Cruz Biotechnology, Inc.) and protease inhibitor cocktail (Sigma-Aldrich; Merck KGaA) on ice. A bicinchoninic acid protein assay kit (Pierce; Thermo Fisher Scientific, Inc.) was used to measure the protein concentration. Equal amounts of the samples $(20 \mu \mathrm{g})$ were separated via $10 \%$ SDS-PAGE. Proteins were transferred to PVDF membranes for $1 \mathrm{~h}$ at $100 \mathrm{~V}$ and blocked with $5 \%$ milk for $1 \mathrm{~h}$ at room temperature. Membranes were then incubated with the primary antibodies [Bcl-2, 1:1,000, cat. no. 3498 and native caspase-3, 1:1,000, cat. no. 9662 (Cell Signaling Technology, Inc.); PTEN, 1:1,000; cat. no. 9188 (Cell Signaling Technology, Inc.] in blocking solutions at $4^{\circ} \mathrm{C}$ overnight before detection with HRP-conjugated secondary antibodies. Immunoreactive bands were visualized on film by enhanced chemiluminescent substrate (Pierce, Thermo Fisher Scientific, Inc.) ImagePro Plus version 7.0 (Media Cybernetics) software was used to calculate the density.

TUNEL staining for $\mathrm{H}_{2} \mathrm{O}_{2}$-mediated apoptosis. After 7 days, cells were placed for $30 \mathrm{~min}$ in culture medium containing a final concentration of $100 \mu \mathrm{M} \mathrm{H}_{2} \mathrm{O}_{2}$. Then, in $4 \%$ paraformaldehyde in PBS for $5 \mathrm{~min}$ at room temperature for fixation. Fragmented DNA in apoptotic cells was quantified using a TUNEL System (Roche Diagnostics) on 96-well plates. TUNEL reagent was added to fixed neurons for $60 \mathrm{~min}$ at $37^{\circ} \mathrm{C}$ in the dark. Fluorescein-12-dUTP-labelled DNA in the cortex was visualized under a fluorescence microscope (magnification, x10, IX71; Olympus Corporation). The nuclei were counterstained with Hoechst 33342 (1 $\mu \mathrm{g} / \mathrm{ml}$; Invitrogen; Thermo Fisher Scientific, Inc.). Hoechst 33342 was added to the neurons for $10 \mathrm{~min}$ at room temperature. For apoptotic cells, positive green fluorescent apoptotic nuclei in three view fields that were selected randomly in each plate were examined. ImagePro Plus version 7.0 image analysis software (Media Cybernetics, Inc.) was used to calculate the percentage of neuronal apoptosis. The average value was taken as the final result.

Statistical analysis. All statistical analyses were performed using SPSS 18.0 software (SPSS, Inc.). All data are presented as the mean \pm standard error of mean, and $\chi^{2}$ test or one-way analysis of variance (ANOVA) was used for comparisons among the four groups. For all ANOVA measures, Tukey's test was used following ANOVA; groups were treated as the independent variable and the levels of the outcome variables as the dependent variables. $\mathrm{P}<0.05$ was considered to indicate a statistically significant difference.

\section{Results}

Culture and identification of forebrain cortical neurons. The procedures of isolation and neuron seeding were completed within $4 \mathrm{~h}$ of animal sacrifice. The plating medium was replaced with serum-free medium $4 \mathrm{~h}$ later. After 7 days of culture, immunocytochemistry was performed to identify the cerebral cortical neurons (Fig. 1). The neurons were immunostained red by $\beta$-tubulin III, a widely used neuron marker (27-29), and their nuclei were stained blue by Hoechst 33342 . The purity of the neurons in the cultures (proportion of $\beta$-tubulin III positive neurons) was $94.78 \pm 1.56 \%$.

Efficiency of specific inhibitors of the PTEN/Akt/mTOR signaling pathway. After 7 days of culture, samples from the four treatment groups were collected for western blot analysis (Fig. 2). The inhibition efficiency of $\mathrm{bpV}$ (pic) was detected, and it was demonstrated that it could effectively inhibit the expression of PTEN (Fig. S1). After adding PTEN inhibitor $\mathrm{bpV}$ (pic), the phosphorylation levels of Akt and p70S6K (Thr421/Ser424) of the bpV(pic) group were increased significantly compared with the control group (Fig. 2). In addition, the phosphorylation levels of mTOR were slightly increased. No clear dose-dependent effects of $b p V(p i c)$ were observed. Neurons were cultured to analyze the inhibitive efficiency of $\mathrm{bpV}$ (pic) at the day 14 time point, and it was demonstrated that the inhibition was still effective. In the LY294002 + bpV(pic) group, the high expression levels of p-Akt and p-p70S6K (Thr421/Ser424) induced by bpV(pic) were eliminated by LY294002. The p-mTOR/mTOR ratio decreased compared with the control group, as LY294002 inhibited the kinase activity of PI3K on mTOR. The levels of total mTOR and Akt were markedly increased in the LY+bpv group compared with control. In the ridaforolimus $+\mathrm{bpV}$ (pic) group, the relative expression of p-Akt was increased; however, the phosphorylation levels of mTOR and p70S6K(Thr421/Ser424) were significantly decreased.

Effects of specific inhibitors on axon growth. The neurons were seeded on a surface coated with PLL + CSPGs + LN . After plating, the medium was replaced with serum-free medium, and the inhibitors were added to the medium. After 10 days of culture, immunocytochemistry was performed to evaluate the neurite initiation and elongation abilities in the presence of CSPGs. Mouse anti-CSPG and rabbit anti- $\beta$-tubulin III antibodies were used to identify CSPG and neurons, respectively. The mean maximum axon lengths were $107.78 \pm 21.95,102.92 \pm 26.51,96.55 \pm 25.37$ and $218.57 \pm 26.77 \mu \mathrm{m}$ in the control, LY294002 + bpV(pic), ridaforolimus $+\mathrm{bpV}$ (pic) and $\mathrm{bpV}$ (pic) groups, respectively (Fig. 3A and B); a significant increase was observed in the bpV(pic) group compared with control (one-way ANOVA, $\mathrm{P}<0.001)$. The number of primary neurites was $3.1 \pm 0.96$, $3.1 \pm 1.05,2.9 \pm 1.40$ and $4.5 \pm 1.90$ in the control, LY294002 $+\mathrm{bpV}($ pic), ridaforolimus $+\mathrm{bpV}($ pic) and $\mathrm{bpV}($ pic) groups, respectively (Fig. 3A and $\mathrm{C}$ ). The number of neurites was significantly increased in the $\mathrm{bpV}(\mathrm{pic})$ group compared with control (one-way ANOVA, $\mathrm{P}<0.001$ ). The percentage of neurites that crossed the CSPG border was $6.32 \pm 2.01 \%$, $6.25 \pm 1.71,7.34 \pm 2.51$ and $20.1 \pm 1.99 \%$ in the four groups (Fig. 4). The percentage of neurites that crossed the CSPG border was significantly increased in the $\mathrm{bpV}$ (pic) group compared with control (one-way ANOVA, $\mathrm{P}<0.01$ ). 


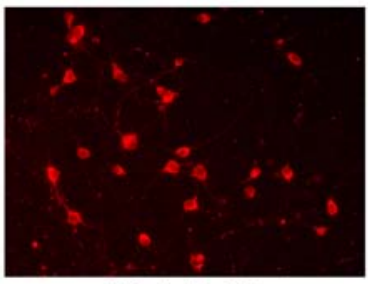

$\beta$-tubulin III

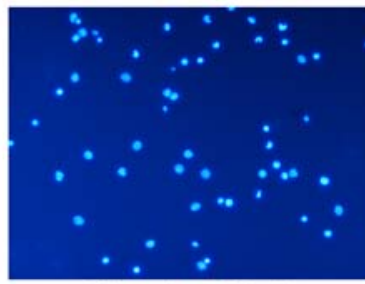

Hoechst 33342

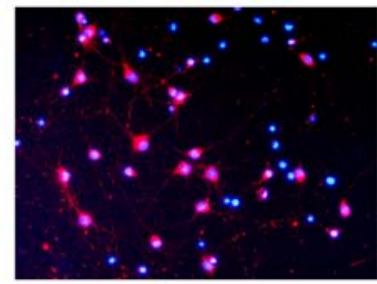

Merge

Figure 1. Neuronal culture and identification. Primary neuronal cultures from the cerebral cortex of Wistar rat pups (postnatal day 0) were stained with Hoechst 33342 (blue) and anti- $\beta$-tubulin III (red). The purity of the neurons in the cultures was $94.78 \pm 1.56 \%$. Magnification, x20.

A

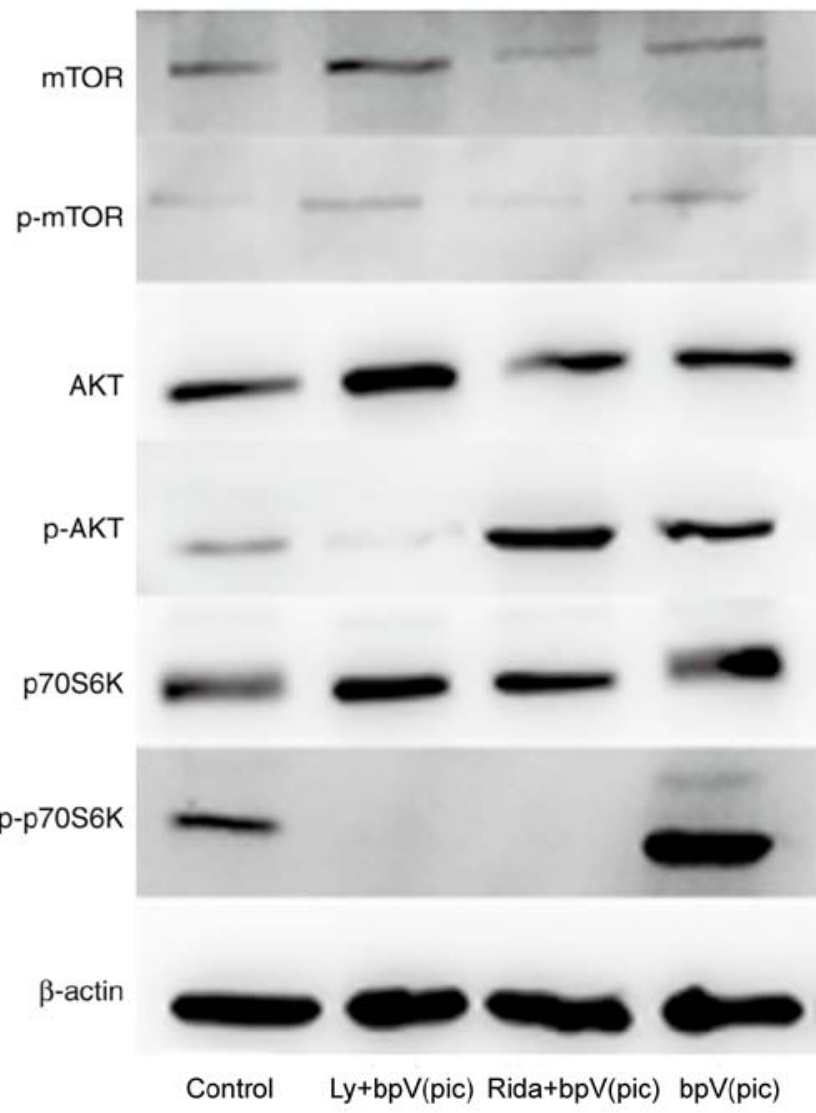

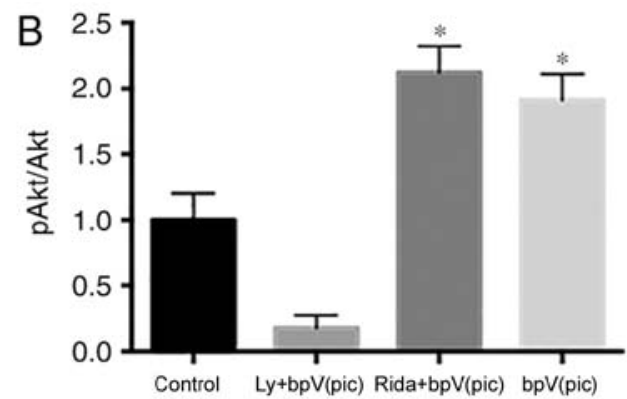
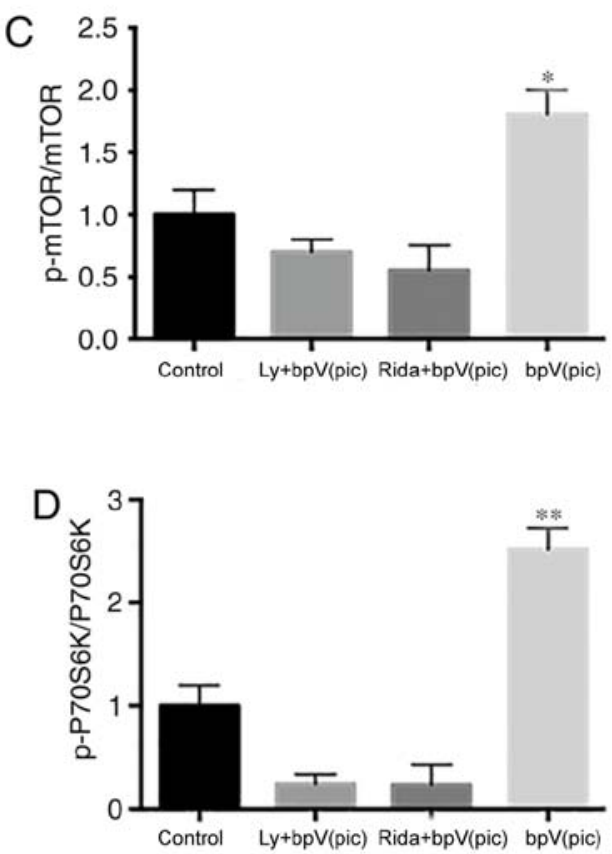

Figure 2. Efficiency of specific inhibitors of the PTEN/Akt/mTOR signaling pathway. (A) Western blot (7 days post-culture) showing the decreased expression of the PTEN/Akt/mTOR signaling pathway. Quantification of (B) p-AKT/AKT, (C) p-mTOR/mTOR and (D) p-p70S6K/p70S6K. "P<0.05 vs. control, ${ }^{* *} \mathrm{P}<0.001$ vs. control. p-, phosphorylated; LY, LY294002; bpV(pic), dipotassium bisperoxo (picolinato) oxovanadate; Rida, ridaforolimus; p70S6K, p70-S6 kinase 1.

Effects of specific inhibitors on $\mathrm{H}_{2} \mathrm{O}_{2}$-mediated apoptosis. After the plating medium was replaced with serum-free medium, the inhibitors were added to the medium. After 7 days of culture, cells were incubated in culture medium containing $100 \mu \mathrm{M} \mathrm{H}_{2} \mathrm{O}_{2}$ for $30 \mathrm{~min}$. Western blotting and TUNEL staining were performed to explore the effects of specific inhibitors on neuronal apoptosis. Using western blotting analysis (Fig. 5), it was determined that Bcl-2 protein exhibited a higher level of expression in the ridaforolimus + $\mathrm{bpV}$ (pic) and $\mathrm{bpV}$ (pic) groups, and reduced expression in the LY294002 + bpV(pic) group compared with the control group.
The expression of caspase-3 was not significantly different across the four groups.

Apoptosis was further analyzed using TUNEL staining; cells and apoptotic cells were stained blue and green, respectively. The percentage of neuronal apoptosis was 49.1 \pm 3.2 , $66.1 \pm 7.9,32.6 \pm 4.0$ and $27.8 \pm 6.3 \%$ in the control, LY294002 $+\mathrm{bpV}(\mathrm{pic})$, ridaforolimus $+\mathrm{bpV}(\mathrm{pic})$ and $\mathrm{bpV}(\mathrm{pic})$ groups, respectively (Fig. 6). The percentage of neuronal apoptosis was significantly reduced in the ridaforolimus $+\mathrm{bpV}(\mathrm{pic})$ and bpV(pic) groups compared with control (one-way ANOVA, $\mathrm{P}<0.05)$. 
A
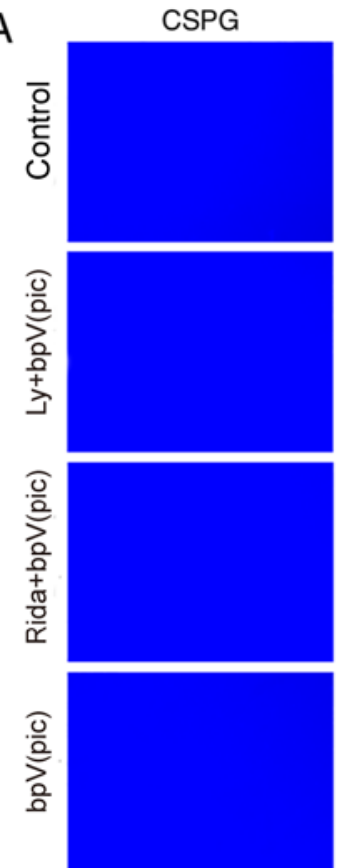

$\beta$-tubulin III
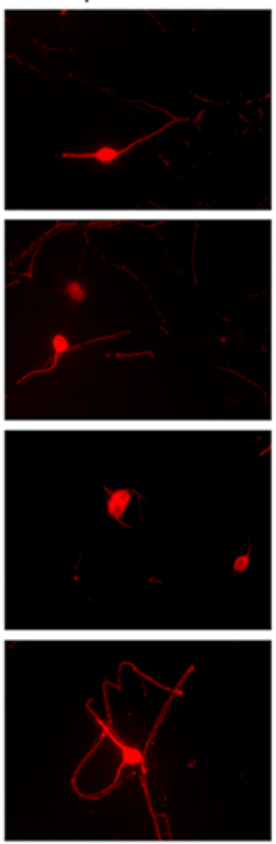
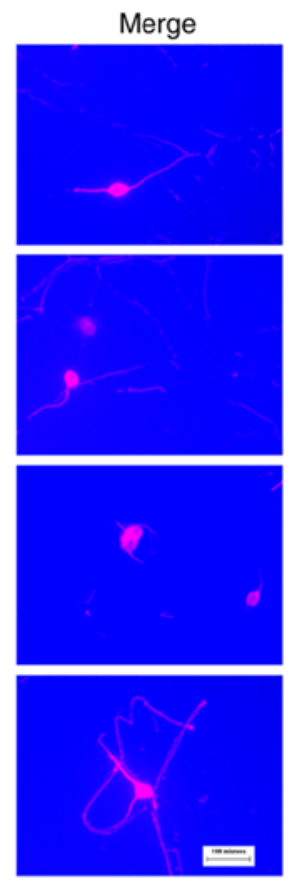
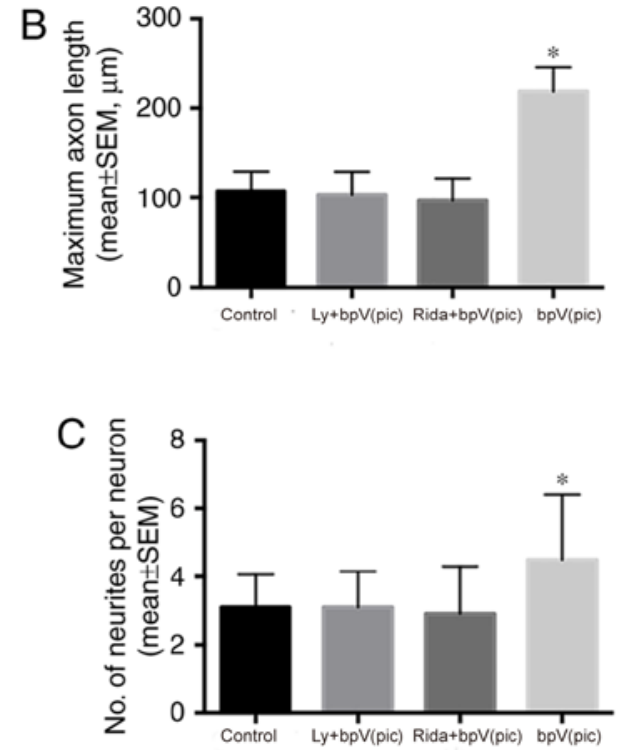

Figure 3. PTEN inhibition promotes neurite elongation and initiation over the chondroitin sulfate proteoglycan substrate in vitro. (A) Different inhibitors were added to the medium; 10 days later, neurons were double stained for $\beta$-tubulin III (red) and CSPGs (blue). The maximum neurite length and number of neurites were measured. Magnification, x40. (B) Quantification of the maximum neurite length. The maximum axon lengths showed a significant increase in the $\mathrm{bpV}$ (pic) group (one-way ANOVA, $\mathrm{P}<0.001$ ). (C) Number of neurites. The number of neurites was significantly higher in the bpV(pic) group (one-way ANOVA, $\mathrm{P}<0.001$ ). "P<0.05 vs. control. ANOVA, analysis of variance; LY/L, LY294002; bpV(pic), dipotassium bisperoxo (picolinato) oxovanadate; Rida/R, ridaforolimus; SEM, standard error of the mean.

A
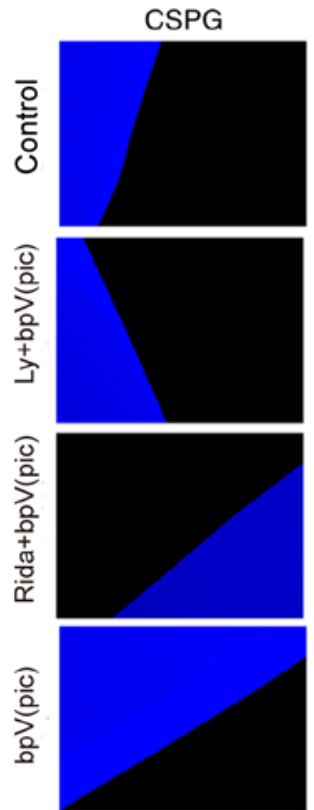

$\beta$-tubulin III
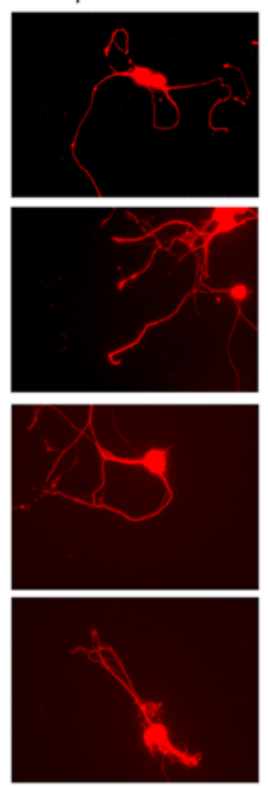
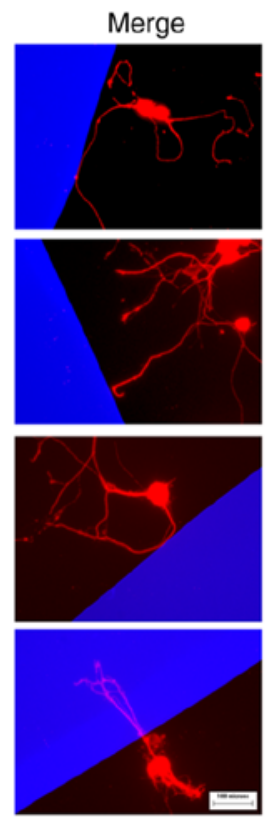

B

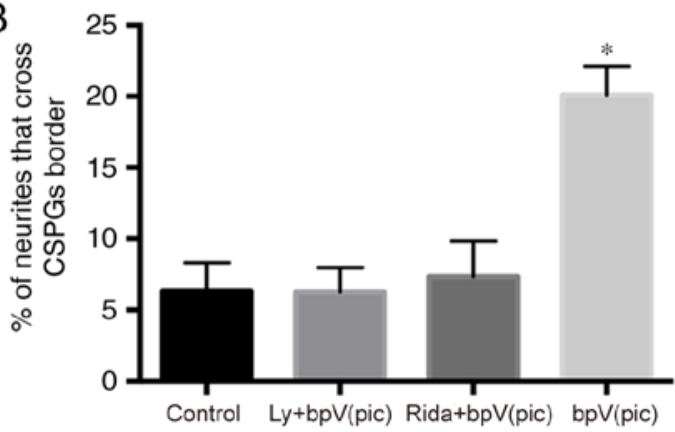

Figure 4. PTEN silencing promotes the ability of neurites to cross the chondroitin sulfate proteoglycan border. (A) Different inhibitors were added to the medium; 10 days later, neurons were double stained for $\beta$-tubulin III (red) and CSPGs (blue). Neurite crossing and contact with the CSPGs border were also measured. the Magnification, x40. (B) Quantification of the percentage of neurites that crossed the CSPG border. The percentage of neurites that crossed the CSPG border was significantly higher in the $\mathrm{bpV}$ (pic) group (one-way ANOVA, $\mathrm{P}<0.01$ ). ${ }^{*} \mathrm{P}<0.05$ vs. control. CSPGs, chondroitin sulfate proteoglycans; bpV(pic), dipotassium bisperoxo (picolinato) oxovanadate; Rida/R, ridaforolimus; LY/L, LY294002.

\section{Discussion}

Studies (15) in the last few decades have focused on characterizing environmental inhibitory molecules in the adult central nervous system. Our previous study (23) showed that an shRNA against PTEN promoted neurite outgrowth of cortical neurons and functional recovery in rats following spinal cord contusion. The present study explored the role of the PTEN/Akt/mTOR 
A

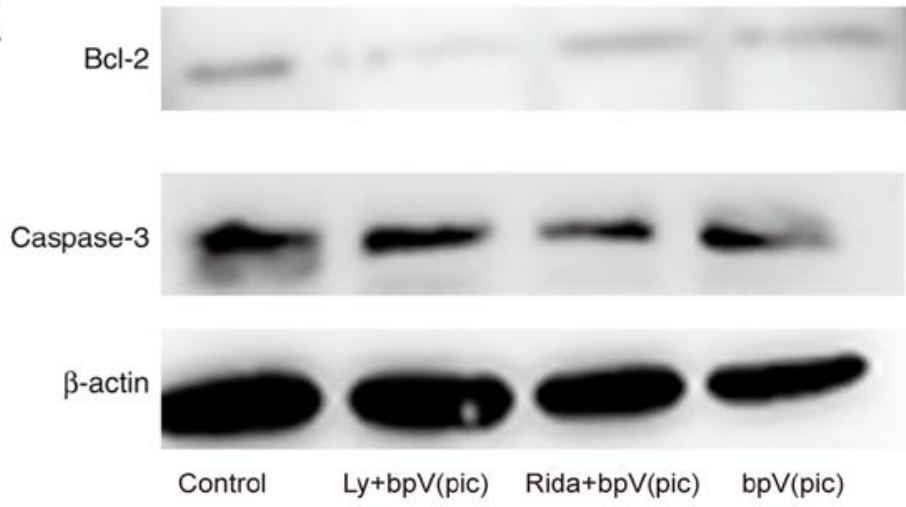

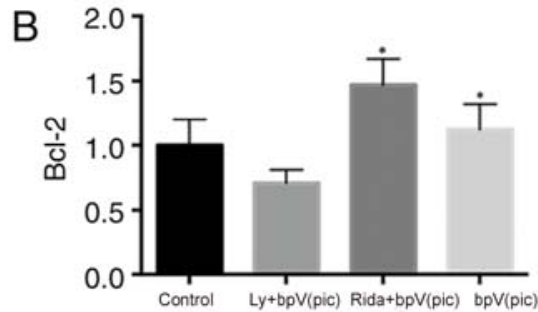

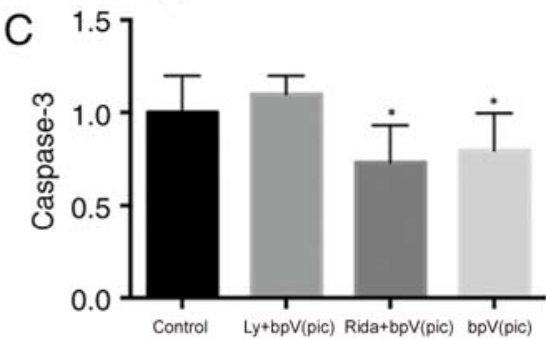

Figure 5. Effects of specific inhibitors on $\mathrm{H}_{2} \mathrm{O}_{2}$-mediated apoptosis. (A) Western blotting (7 days post-culture) showing the expression of (B) Bcl-2 and (C) caspase-3. "P<0.05 vs. Control. LY/L, LY294002; bpV(pic), dipotassium bisperoxo (picolinato) oxovanadate; Rida/R, ridaforolimus.

A
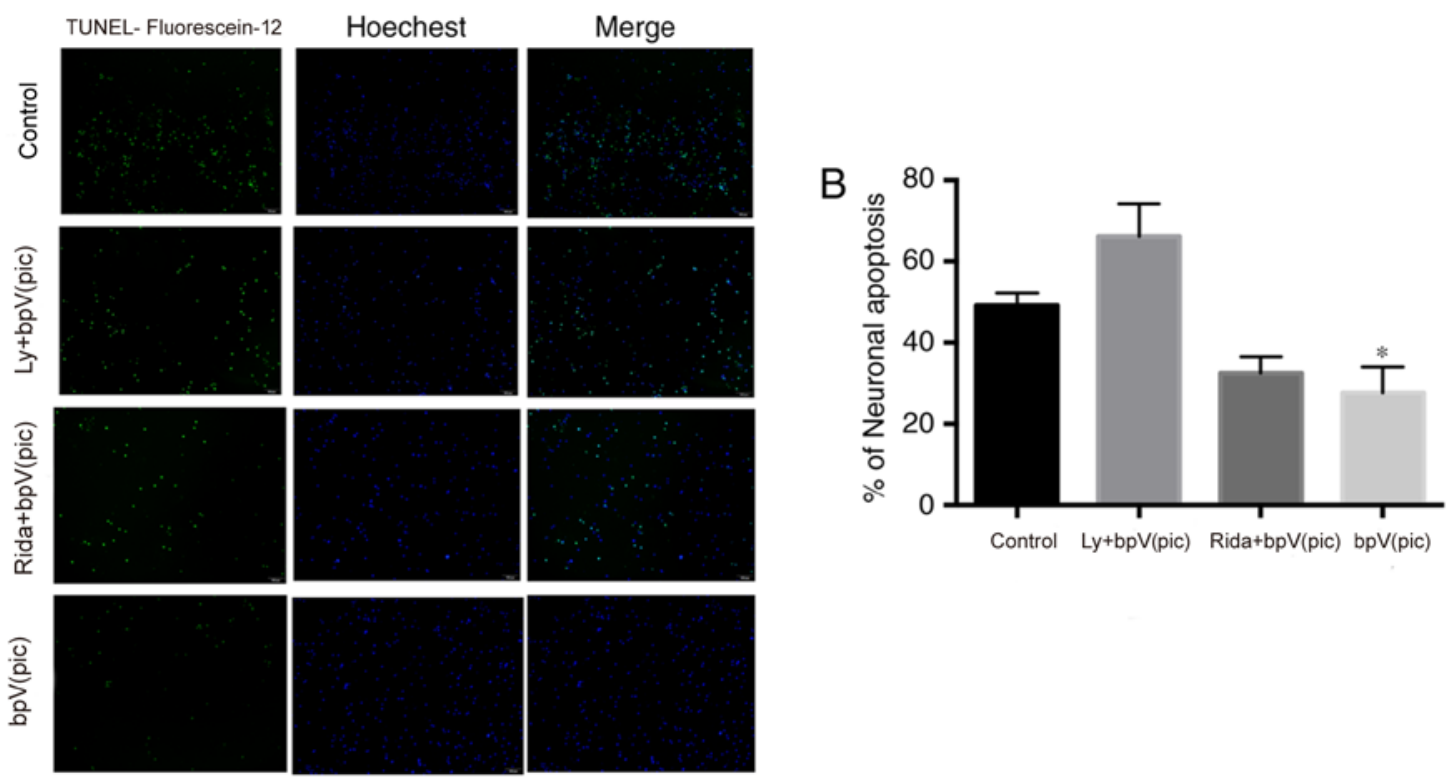

Figure 6. Effects of specific inhibitors on $\mathrm{H}_{2} \mathrm{O}_{2}$-mediated apoptosis. TUNEL staining was performed. (A) Different inhibitors were added to the medium; 7 days later, neurons were double-stained with TUNEL-Fluorescein-12 (green) and Hoechst 33342 (blue). Magnification, x10. (B) Quantification of neuronal apoptosis. LY, LY294002; Rida, ridaforolimus; bpV(pic), dipotassium bisperoxo (picolinato) oxovanadate. * $\mathrm{P}<0.05$ vs. control.

signaling pathway in axonal growth/regeneration and neuronal apoptosis in the presence of CSPGs-mediated inhibition.

PTEN is an important potent tumor suppressor and has been found to be mutated in the development of various cancers, including hepatocellular carcinoma and lung cancer $(30,31)$. It not only serves an essential role in cell proliferation, differentiation, growth and migration, but also affects apoptosis (32-35). According to previous studies $(36,37)$, PTEN dramatically enhances the intrinsic growth/regenerative ability of corticospinal neurons to promote the extension of injured corticospinal tract (CST) axons following SCI with PTEN inactivation. It negatively regulates intracellular levels of PIP3; this dephosphorylation (p-mTOR dephosphorylated into mTOR) is important as it results in inhibition of the Akt signaling pathway (20). The PI3K/Akt signaling pathway has a central role in cell growth and survival through the phosphorylation and inhibition of a number of vital substrates. For example, Akt can activate the transcription of the proapoptotic genes encoding Fas receptor and Bcl-2-like protein 11, and inactivate the proapoptotic Bcl-2 family member Bcl-2-associated agonist of cell death (20,38-40). mTOR is a serine/threonine protein kinase that consists of two types of protein complexes (41). The protein kinase mTOR phosphorylates multiple downstream proteins of the PI3K/Akt pathway (41). In this experiment, p70S6K was selected as a biomarker of mTOR activity (41).

The present study used pharmacological methods. The specific inhibitors of PTEN, Akt and mTOR, 
bpV(pic), LY294002 and ridaforolimus, were used to treat cells $(10,42,43)$. The experiments comprised four groups based on the inhibitor used in each. Western blot analysis was used to verify the efficiency of the specific inhibitors. Compared with the control group, the phosphorylation levels of Akt, p70S6K and mTOR of the $\mathrm{bpV}$ (pic) group were significantly increased. This suggested that $\mathrm{bpV}(\mathrm{pic})$ had an effect on the PTEN/Akt/mTOR signaling pathway. When LY294002 was added, the phosphorylation levels of Akt, mTOR and p70S6K were downregulated. When the ridaforolimus was added, the phosphorylation levels of mTOR and p70S6K were downregulated, but the level of p-Akt was unchanged. These results demonstrated that these specific inhibitors exhibited high inhibitory efficiency, and that the $\mathrm{PI} 3 \mathrm{~K} / \mathrm{Akt} / \mathrm{mTOR}$ signaling pathway was activated in cells following PTEN inhibition.

Axonal regeneration and sprouting are two important strategies for SCI repair (8). In addition, they may be central to promoting reinnervation and functional recovery after SCI. It was first explored whether cerebral cortical neurons would show significant improvement in overcoming CSPGs-mediated axonal inhibition following PTEN inhibition. The maximum axon length, number of neurites and percentage of neurites that crossed the CSPGs border were all significantly higher in the $\mathrm{bpV}$ (pic) group. The neurons gained an increased ability to extend their axons over the CSPGs substrate and to cross into the CSPGs-rich regions. This finding is consistent with our and other previous studies $(8,23,44)$. In addition, when LY294002 and ridaforolimus were added, the ability for neuronal regeneration was attenuated. This suggested that the PTEN inhibition promoted axonal growth/regeneration through the PI3K/Akt/mTOR signaling pathway. Then, the $\mathrm{H}_{2} \mathrm{O}_{2}$-induced apoptosis of cerebral cortical neurons following PTEN inhibition was explored. The results of TUNEL staining showed that the percentage of neuronal apoptosis was significantly lower in the ridaforolimus $+\mathrm{bpV}$ (pic) and $\mathrm{bpV}$ (pic) groups. However, it was higher in the LY294002 + bpV(pic) group, suggesting that PI3K signaling was critically involved in apoptotic induction. In addition, mTOR appeared to not be a critical downstream target; based on previous findings, glycogen synthase kinase $3 \beta$ may be an important downstream target for neuronal apoptosis $(1,45)$.

SCI is a complicated pathophysiological process that involves a cascade of cellular and biochemical events (2,46-48). The present study found that the PI3K/Akt/mTOR signaling pathway served an important role in neuronal regeneration. Whether other signaling pathways are involved in neuronal regeneration, and whether there is crossover activity between these signaling pathways, requires further investigation. In any case, a single treatment strategy, in spite of decreases in the inhibitory factors present in the microenvironment or the promotion of neuron-intrinsic regenerative abilities, is not sufficient to support neuronal regeneration; thus, combining treatments with other successful strategies targeting different mechanisms for repair may be more successful. Further studies should be performed to address the issues associated with SCI.

$\mathrm{bpV}($ pic) is an effective PTEN inhibitor. PTEN inhibition mediated by $\mathrm{bpV}(\mathrm{pic})$ promoted the axonal elongation and initiation abilities of cerebral cortical neurons, as well as the ability of these axons to cross the CSPG border; these effects were mediated via the PTEN/PI3K/Akt/mTOR signaling pathway. Furthermore, PTEN inhibition mediated by bpV(pic) protected neurons from apoptosis. The results of the present study may provide a potential novel strategy for the treatment of SCI through PTEN inhibition.

\section{Acknowledgements}

The authors would like to thank Dr Jianming Yang (Key Laboratory of Immuno Microenvironment and Disease of the Educational Ministry of China, Department of Immunology, Tianjin Medical University, Tianjin), for her help in writing.

\section{Funding}

This work was supported by the National Natural Science Foundation of China (grant no. 81501899), the State Key Program of the National Natural Science Foundation of China (grant no. 81330042), the Special Program for Sino-Russian Joint Research Sponsored by the Ministry of Science and Technology, China (grant no. 2014DFR31210), the Key Program Sponsored by the Tianjin Science and Technology Committee, China (grant nos. 13RCGFSY19000 and 14ZCZDSY00044), the Science Foundation of Tianjin Medical University for Young Scholars (grant no. 2014KYQ01) and the Science Foundation of Tianjin Medical University General Hospital for Young Scholars (grant no. ZYYFY2014037).

\section{Availability of data and materials}

The datasets used and/or analyzed during the current study are available from the corresponding author on reasonable request.

\section{Authors' contributions}

XK and SF conceived and designed the study. SL, JJ, HZ and $\mathrm{CZ}$ performed the experiments. These four authors contributed equally. LLiu and JL analyzed the data and drafted the Abstract and Introduction; LLu, XL and CZ interpreted the data and wrote the remaining parts of this manuscript; YK and YL performed the secondary data analyses and revised the manuscript for intellectual and scientific content, and ZC and YR contributed to the conception of the study. All authors read and approved the manuscript.

\section{Ethics approval and consent to participate}

All experimental procedures involving animals were approved by the Ethics Committee of Tianjin Medical University.

\section{Patient consent for publication}

Not applicable.

\section{Competing interests}

The authors declare that they have no competing interests. 


\section{References}

1. Jain NB, Ayers GD, Peterson EN, Harris MB, Morse L, O'Connor KC and Garshick E: Traumatic spinal cord injury in the United States, 1993-2012. JAMA 313: 2236-2243, 2015.

2. Blesch A and Tuszynski MH: Spinal cord injury: Plasticity, regeneration and the challenge of translational drug development. Trends Neurosci 32: 41-47, 2009.

3. Sun F and He Z: Neuronal intrinsic barriers for axon regeneration in the adult CNS. Curr Opin Neurobiol 20: 510-518, 2010.

4. Yang $\mathrm{P}$ and Yang Z: Enhancing intrinsic growth capacity promotes adult CNS regeneration. J Neurol Sci 312: 1-6, 2012.

5. Cao L, Zhu YL, Su Z, Lv B, Huang Z, Mu L and He C: Olfactory ensheathing cells promote migration of Schwann cells by secreted nerve growth factor. Glia 55: 897-904, 2007.

6. Fouad K, Schnell L, Bunge MB, Schwab ME, Liebscher T and Pearse DD: Combining Schwann cell bridges and olfactory-ensheathing glia grafts with chondroitinase promotes locomotor recovery after complete transection of the spinal cord. J Neurosci 25: 1169-1178, 2005.

7. Harel NY and Strittmatter SM: Can regenerating axons recapitulate developmental guidance during recovery from spinal cord injury? Nat Rev Neurosci 7: 603-616, 2006.

8. Klapka N and Müller HW: Collagen matrix in spinal cord injury. J Neurotrauma 23: 422-435, 2006.

9. Paveliev M, Lume M, Velthut A, Phillips M, Arumäe U and Saarma M: Neurotrophic factors switch between two signaling pathways that trigger axonal growth. J Cell Sci 120: 2507-2516, 2007.

10. Schwab ME: Functions of Nogo proteins and their receptors in the nervous system. Nat Rev Neurosci 11: 799-811, 2010.

11. Silver J and Miller JH: Regeneration beyond the glial scar. Nat Rev Neurosci 5: 146-156, 2004.

12. Liu K, Tedeschi A, Park KK and He Z: Neuronal intrinsic mechanisms of axon regeneration. Annu Rev Neurosci 34: 131-152, 2011.

13. Sun F, Park KK, Belin S, Wang D, Lu T, Chen G, Zhang K, Yeung C, Feng G, Yankner BA and He Z: Sustained axon regeneration induced by co-deletion of PTEN and SOCS3. Nature 480: 372-375, 2011

14. Busch SA and Silver J: The role of extracellular matrix in CNS regeneration. Curr Opin Neurobiol 17: 120-127, 2007.

15. Yiu G and He Z: Glial inhibition of CNS axon regeneration. Nat Rev Neurosci 7: 617-627, 2006.

16. Park KK, Liu K, Hu Y, Kanter JL and He Z: PTEN/mTOR and axon regeneration. Exp Neurol 223: 45-50, 2010.

17. Shin JE, Cho Y, Beirowski B, Milbrandt J, Cavalli V and DiAntonio A: Dual leucine zipper kinase is required for retrograde injury signaling and axonal regeneration. Neuron 74 : 1015-1022, 2012.

18. Smith PD, Sun F, Park KK, Cai B, Wang C, Kuwako K, Martinez-Carrasco I, Connolly L and He Z: SOCS3 deletion promotes optic nerve regeneration in vivo. Neuron 64: 617-623, 2009.

19. Maehama Tand Dixon JE: The tumor suppressor,PTEN/MMAC1, dephosphorylates the lipid second messenger, phosphatidylinositol 3,4,5-trisphosphate. J Biol Chem 273: 13375-13378, 1998.

20. Sansal I and Sellers WR: The biology and clinical relevance of the PTEN tumor suppressor pathway. J Clin Oncol 22: 2954-2963, 2004.

21. Stambolic V, Suzuki A, de la Pompa JL, Brothers GM, Mirtsos C, Sasaki T, Ruland J, Penninger JM, Siderovski DP and Mak TW: Negative regulation of PKB/Akt-dependent cell survival by the tumor suppressor PTEN. Cell 95: 29-39, 1998.

22. Park KK, Liu K, Hu Y, Smith PD, Wang C, Cai B, Xu B, Connolly L, Kramvis I, Sahin M and He Z: Promoting axon regeneration in the adult CNS by modulation of the PTEN/mTOR pathway. Science 322: 963-966, 2008.

23. Zhou H, Li X, Wu Q, Li F, Fu Z, Liu C, Liang Z, Chu T, Wang T, Lu L, et al: shRNA against PTEN promotes neurite outgrowth of cortical neurons and functional recovery in spinal cord contusion rats. Regen Med 10: 411-429, 2015.

24. Zimmermann M: Ethical principles for the maintenance and use of animals in neuroscience research. Neurosci Lett 73: 1, 1987.

25. Tom VJ, Steinmetz MP, Miller JH, Doller CM and Silver J: Studies on the development and behavior of the dystrophic growth cone, the hallmark of regeneration failure, in an in vitro model of the glial scar and after spinal cord injury. J Neurosci 24: $6531-6539,2004$

26. Jordan PM, Ojeda LD, Thonhoff JR, Gao J, Boehning D, Yu Y and Wu P: Generation of spinal motor neurons from human fetal brain-derived neural stem cells: Role of basic fibroblast growth factor. J Neurosci Res 87: 318-332, 2009.
27. Geisert EE Jr and Frankfurter A: The neuronal response to injury as visualized by immunostaining of class III beta-tubulin in the rat. Neurosci Lett 102: 137-141, 1989.

28. Ignatova TN, Kukekov VG, Laywell ED, Suslov ON, Vrionis FD and Steindler DA: Human cortical glial tumors contain neural stem-like cells expressing astroglial and neuronal markers in vitro. Glia 39: 193-206, 2002.

29. Kempermann G, Gast D, Kronenberg G, Yamaguchi M and Gage FH: Early determination and long-term persistence of adult-generated new neurons in the hippocampus of mice. Development 130: 391-399, 2003.

30. Li DM and Sun H: TEP1, encoded by a candidate tumor suppressor locus, is a novel protein tyrosine phosphatase regulated by transforming growth factor beta. Cancer Res 57: 2124-2129, 1997.

31. Steck PA, Pershouse MA, Jasser SA, Yung WK, Lin H, Ligon AH, Langford LA, Baumgard ML, Hattier T, Davis T, et al: Identification of a candidate tumour suppressor gene, MMAC1, at chromosome $10 \mathrm{q} 23.3$ that is mutated in multiple advanced cancers. Nat Genet 15: 356-362, 1997.

32. Alexiou GA and Voulgaris S: The role of the PTEN gene in malignant gliomas. Neurol Neurochir Pol 44: 80-86, 2010.

33. Goberdhan DC and Wilson C: PTEN: Tumour suppressor, multifunctional growth regulator and more. Hum Mol Genet $12 \mathrm{Spec}$ No 2: R239-R248, 2003.

34. Leslie NR, Maccario H, Spinelli L and Davidson L: The significance of PTEN's protein phosphatase activity. Adv Enzyme Regul 49: 190-196, 2009.

35. Shi Y, Paluch BE, Wang X and Jiang X: PTEN at a glance. J Cell Sci 125: 4687-4692, 2012

36. Liu K, Lu Y, Lee JK, Samara R, Willenberg R, Sears-Kraxberger I, Tedeschi A, Park KK, Jin D, Cai B, et al: PTEN deletion enhances the regenerative ability of adult corticospinal neurons. Nat Neurosci 13: 1075-1081, 2010.

37. Zukor K, Belin S, Wang C, Keelan N, Wang X and He Z: Short hairpin RNA against PTEN enhances regenerative growth of corticospinal tract axons after spinal cord injury. J Neurosci 33 : 15350-15361, 2013.

38. Brunet A, Bonni A, Zigmond MJ, Lin MZ, Juo P, Hu LS, Anderson MJ, Arden KC, Blenis J and Greenberg ME: Akt promotes cell survival by phosphorylating and inhibiting a Forkhead transcription factor. Cell 96: 857-868, 1999.

39. Datta SR, Dudek H, Tao X, Masters S, Fu H, Gotoh Y and Greenberg ME: Akt phosphorylation of BAD couples survival signals to the cell-intrinsic death machinery. Cell 91: 231-241, 1997.

40. Dijkers PF, Medema RH, Pals C, Banerji L, Thomas NS, Lam EW, Burgering BM, Raaijmakers JA, Lammers JW, Koenderman L and Coffer PJ: Forkhead transcription factor FKHR-L1 modulates cytokine-dependent transcriptional regulation of p27(KIP1). Mol Cell Biol 20: 9138-9148, 2000.

41. Park KK, Hu Y, Muhling J, Pollett MA, Dallimore EJ, Turnley AM, Cui Q and Harvey AR: Cytokine-induced SOCS expression is inhibited by cAMP analogue: Impact on regeneration in injured retina. Mol Cell Neurosci 41: 313-324, 2009.

42. Schmid AC, Byrne RD, Vilar R and Woscholski R: Bisperoxovanadium compounds are potent PTEN inhibitors. FEBS Lett 566: 35-38, 2004

43. Vlahos CJ, Matter WF, Hui KY and Brown RF: A specific inhibitor of phosphatidylinositol 3-kinase, 2-(4-morpholinyl)8-phenyl-4H-1-benzopyran-4-one (LY294002). J Biol Chem 269: 5241-5248, 1994.

44. Lu Y, Belin S and He Z: Signaling regulations of neuronal regenerative ability. Curr Opin Neurobiol 27: 135-142, 2014.

45. Dill J, Wang H, Zhou F and Li S: Inactivation of glycogen synthase kinase 3 promotes axonal growth and recovery in the CNS. J Neurosci 28: 8914-8928, 2008 .

46. Bartus K, James ND, Bosch KD and Bradbury EJ: Chondroitin sulphate proteoglycans: Key modulators of spinal cord and brain plasticity. Exp Neurol 235: 5-17, 2012

47. Gervasi NM, Kwok JC and Fawcett JW: Role of extracellular factors in axon regeneration in the CNS: Implications for therapy. Regen Med 3: 907-923, 2008.

48. Jiang H, Guo W, Liang X and Rao Y: Both the establishment and the maintenance of neuronal polarity require active mechanisms: Critical roles of GSK-3beta and its upstream regulators. Cell 120: 123-135, 2005.

This work is licensed under a Creative Commons Attribution-NonCommercial-NoDerivatives 4.0 International (CC BY-NC-ND 4.0) License. 